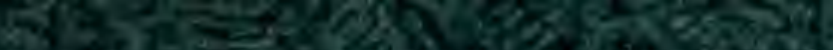

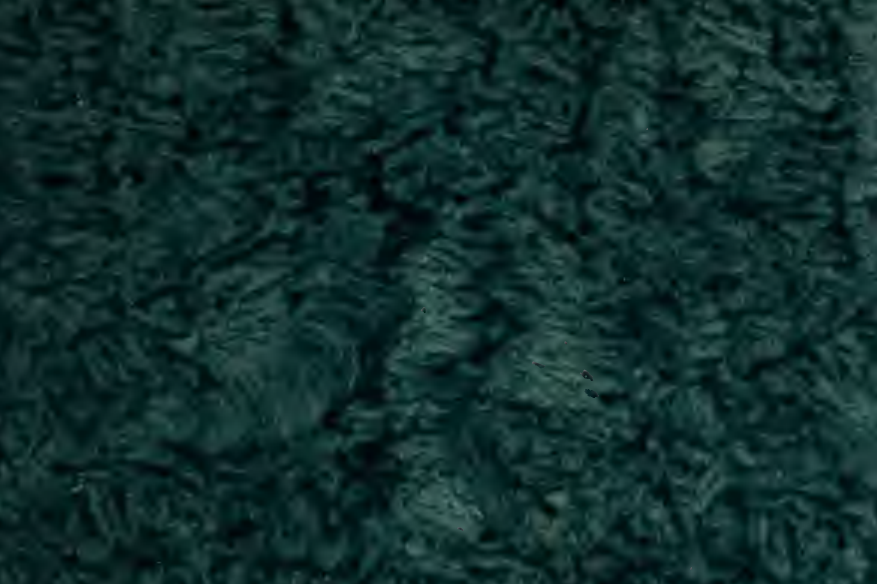

\title{
2.
}

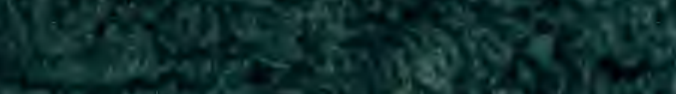

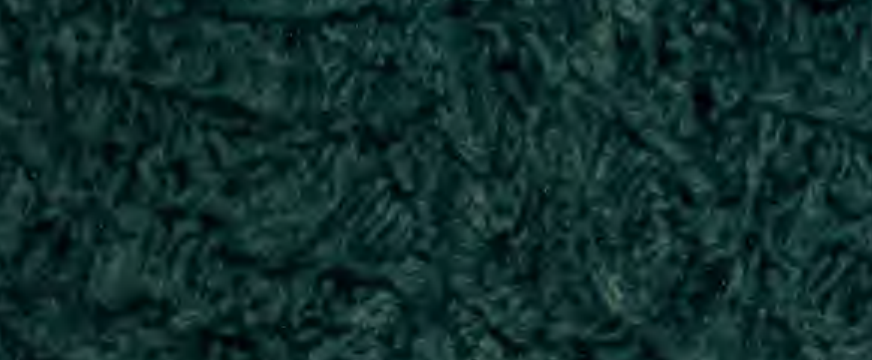
(5)

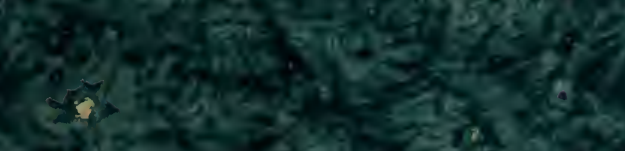

W.

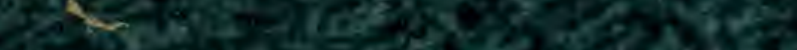

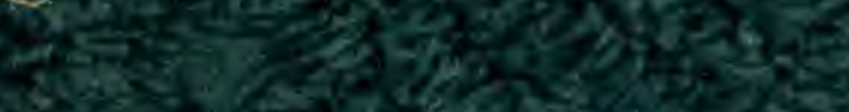

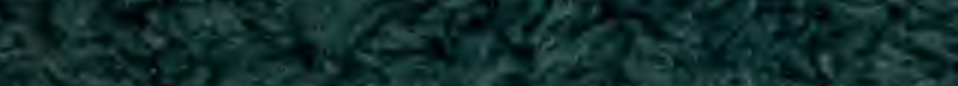
W.

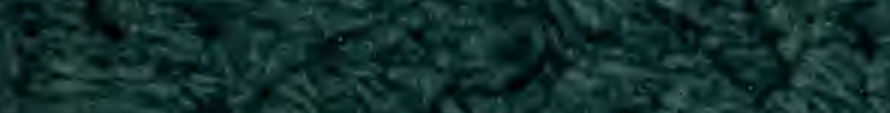

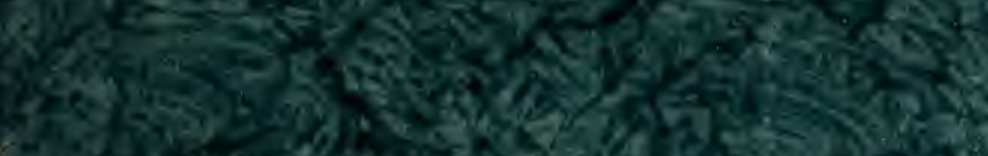

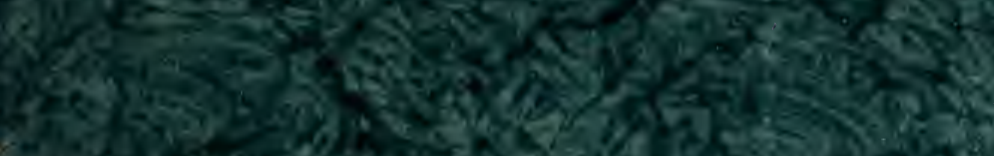

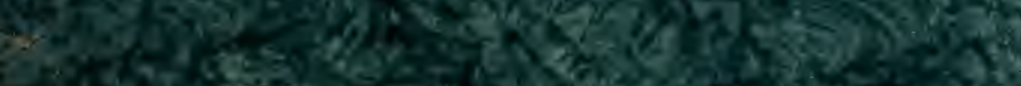

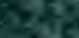





\section{PRÉCIS HISTORIQUE}

DE L' ÉCONOMIE RURALE

\section{DES C H I NOIS,}

Préfenté à $l$ Académie Royale des Sciences de Suède l'an. 1754., par M. Charles Guftave Eckeberg, Capitaine d"un vaifSeau de la Compagnie Suédoise des Indes Orientales, publie par Mo Linnaeus, of traduit du Suédois par $M_{\circ}$ Dominique de Blackford.

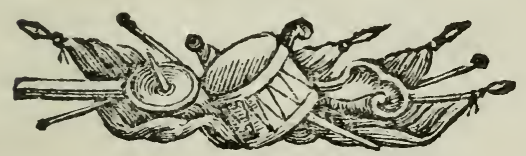

A MILAN. MDCCLXXI.

Chez les Freres Reycends Libraires fous les Arcades de Figini. Avec apprabation. 



\section{A V E R T IS S E M E N T}

\section{DU TRALUCTEUR .}

LE petit ouvrage, dont on offre aujourd'bui la traduction au public, a paru à Stockbolm l'an 1757. Il ef entièrement nouveau dans fon genre: on s'appercoit aifément, que l'Auteur $n$ 'eft pas un voyageur ordinaire, o qu'il a vu avec fruit. Il Seroit d̀ Soubaiter cependant, qu'il eut donné des explications plus parfaites de quelques productions naturelles. Le Traducteur qui eft étranger, n'a eu d' autre but, que de rendre fidèlement fon original \& d'être intelligible. 


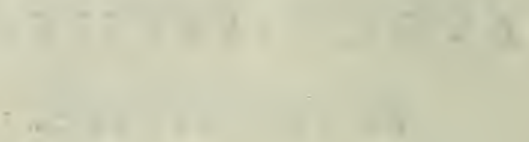

1.

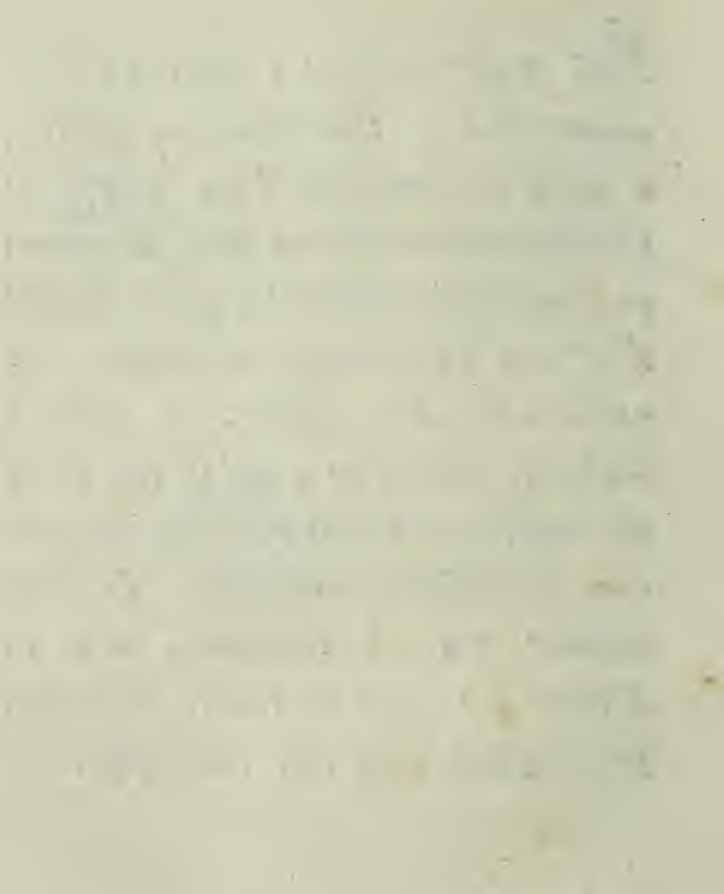




\section{PRÉCIS HISTORIQUE}

DE L'ÉCONOMIE RURALE

\section{DES CH I N O S .}

W L n'y a peut-être point de peuple, qui puiffe fe vanter d'être affès riche en productions naturelles \& de les porféder dans une variété affès graude, pour pouvoir le paffer entièrement des productions étrangères. C'eft cette défectuofité, qui paroit être le lien général qui unit enfemble les fociétés civiles: mais la nature femble avoir fuivi une règle différente à l'égard de la Chine; elle peut fe fuffire à elle même.

Ce pais eft fi heureufement fitué, que les habitans de la partie feptentrionale is plaignent auffi peu du grand froid, que ceux qui habitent la partie méridionale, fe plaignent d'une trop grande chaleur. Le climat des païs intermédiaires eft doux, égal \& il eft par conféquent agréable pour la vie, commode à la fanté \& propre à produire toute forte de plantes.

Les vents alifés, qui font propres à la partie méridionale \& chaude apportent avec euis de grands avantages à ce climat, le vent du nord purifie l'air, diffipant toutes les 
vapeurs pernicieufes, que la chaleur élève fur l'atmolphère, \& le vent du fuł tempère l'éffervefcence de la faifon chaude.

La plus grande partie des frontières de la Chine eft mouillée par de vaftes mers, qui de diftance en diftance forment des golphes, où l'on eft entièrement à l'abri de tempêtes. Comme la nature femble avoir borné ici la navigation, elle lui a ouvert des nouveaux chemins par des rivières \& des fleuves navigables qui fe répandent dans l'intérieur du païs.

Le flux \& le reflux de l'eau, qui s'étend fort avant dans le païs \& cinq milles Suedois au delà de la ville de Canton, facilite la navigation, \& fait que les différentes conzrées peuvent fe communiquer mutuellement leurs productions; ce qui augmente le commerce \& la confommation générale.

Le fol eit fi fertile, que quelque mauvais que puiffent ètre les marais \& les hauteurs, ils récrimpentent cependant abondanment le travail des laboureurs; car le bled, les fruits de la terre \& des arbres, qui mûriffent ici parfaitement \& dans ure variété infinie, s'éfforcent, pcur ainfi dire, à l'envi de combler leurs cultivateurs de conftantes moiffons.

Les grandes \& valtes forêts fourniffent, outre une quantité immenfe de bois de charpente \& d"autres bois propres à bien des nlages, plufieurs efpèces de bois fins \& pré- 
cieux, des fucs utiles, des gommes, des écorces \& des feuilles. Ces forêts font d'ailleurs habitées par un grand nombre de bêtes fauvages qui fervent à la nourriture \& à l'habillement. Des métaux, ditrérentes efpèces de pierre $\&$ de terre, des fels, du fable d'or, des perles, du corail, quoiqu' il ne foit pas de la meilleure qualité \& une infinité d'efpèces de poiffons que le rivage frais \& falé attire en quantité, font voir que la nature ne les a pas traité en marâtre non plus de ce côtè là. La volaille, qu' on trouve ici partout en troupes fort nombreufes, flatte la vue, les oreilles \& le gout. En un mot le regne de la nature eft dans la plus grande perfection à la Chine : elle offre les perfpectives les plus magnifiques, des fituations \& des commodités de toute elpèce, aux quelles ni l'invention ni l'art ne pourroit rien ajouter; \& fi l'on excepte le fuperflu, dont on peut fe paffer, toutes les chofes néceffaires à la nourriture \& à l'habillement, fans qu'on ait beloin, d'avoir recours à l'étranger.

Comme le bien être d'un païs dépend principalement du bon ordre \& de l'indultrie de fes habitans, on peut dire que la Chine jouit particulièrement de ces avantages. Tous ceux qui ont fait des defcriptions de ce paîs ont remarqué l'indultrie des Chinois \& leur dextérité pour toutes fortes de métiers, \& nous en voyons des preuves par les différen- 


\section{4

tes marchandifes, que nos vaiffeaux y voni chercher. Leur païs produit en quantité fuffifante les matières crues.

Je me fuis propofé, pour prouver l'induftrie fingulière des Chinois, de raconter ici brièvement ce que j'ai oblervé pendant ua féjour de quinze mois que i'y ai fait en trois fois différentes, touchant quelques détails de leur économie ordinaire \& habituelle.

\section{De I'Agriculture.}

7 Ans les parties méridionales de la Chi1) ne, qui font frtuées fur la mer, le ris, efpèce de bled, qui profpère le mieux dans un fol bas \& humide, elt comme prefque chés tous les orientaux la principale nourriture. Il eft des efpèces de ris, qui profpèrent dans un fol fec \& haut, comme l'on voit par ci par là dans l'isle de Java, \& d'autres terres hautes. Les provinces Chinoifes qui ont un fol fec \& inézal, fe fervent de ce ris: mais en Quantung ou dans les provinces méridionales qui font baffes, on le femeroit avec perte, parceque fon grain elt petit, qu'il demande le double du tems pour mûrir, au lieu qua le grain de l'autre ris eft plus gros, qu'il croît mieux \&c plus vîte, \& qu'il peut toujours être fous l'eau, fans être endommagé. De cette efpèce il y en a une forte inférieure, qui elt rougeâtre $\& z$ 
dont les gens du commun fe fervent. on s'en fert auffi, pour en faire une eau de vie qu'ils appellent Samfou.

On m'a dit qu'à mefure qu'on avançoit dans la Chine, du midi au feptentrion, la culture du ris diminuoit, \& qu' on cultivoit à proportion plus de feigle, d'orge, de froment, de fèves, de pois \&c. \& que dans les provinces les plus feptentrionales, où le ris re prend pas du tout, on entendoit parfaitement bien la culture du blé.

Néanmoins on plante auffi du froment, des fèves, des petits pois \& des lentilles dans les provinces méridionales, dont les habitans fe fervent en partie pour eux, \& en partic pour les étrangers: Mais on fème beatcoup plus fréquemment du ris, dont je parlerai plus particulièrement, puifqu'on s'en fert aus environs de Canton au lieu de pain.

On f̧̧ait que la Chine eft un païs extrêmement peuplé. La plupart des provinces le font au point, qu'on eft étonné de voir que ce païs puiffe produire affès de blé pour tant de millions d'habitans, d'autant plus qu'à l'exception de quelques rounques ( ${ }^{*}$ ) venant de la Cochinchine $\&$ de quelques vaiffeaux hollandois chargés de blé, ce qui $n$ 'arrive encore que rarement, ils n'en tirent A 3

(*) Efpéces de navires fort grands . 
point de l'étranger : mais quand on fait attention à leur induftrie prefqu' incroyable à cultiver \& à tirer parti de tout, à leur parcimonie \& extrême fobriété dans la vie ordinarie, on eft convaincu qu' un païs qui a des pareils habitans, quelqu'en foit le nombre, ne peut prefque jamais manquer de leur fournir la nourriture néceffaire: au contraire c'elt plutot la multitude de gens laborieux, qui contribue à la richeffe du païs $\&$ à la meilleure fubfiftance des habitans; car chaque agricole laborieux tire toujours plus du fein reconnoiffant de la terre, qu' il n'en peut confommer lui feul.

Le degré de perfection, où l'on a pouffé dans la Chine l'agricuiture \& particulièrement. la culture du ris, eft le principal fondement de la félicité, dont jouit ce paìs. L'écunomie rurale eft auff la profefion, qu' on y honore, \& qu' on y encourage le plus. Les empereurs mêmes pour mieux marquer combien ils eftiment cette profeffion \& pour donner à leurs fujets un exemple digne d'être imité, vont à la campagne à un certain jour de chaque année, accompagnés des grands de leur cour, prennent la charue à la main, préparent \& sèment un diftrict, \& moiffonnent enfuite de leur propre main le fruit de leur travail: mais il faut, que ie me borne aux côtes de Canton. 


\section{Du terrain.}

T E terrein aux environs de Canton eft L auffi varié eú egard à fa fituation, que daus d'autres endroits; tous les lieux bas fon: couverts de terre graffe $\&$ noire: mais à mefure que le terrain s'élève, il fe revêt ' une terre jaune \& rougeâtre melée d'ochr: \& le fable \& le gravier s'y trouvent a plus grande quantité. Lorique cette terre :efte incuite pendant quelque tems, l'altenative de la pluie \& du foleil lui donn une furface, pour ainfi dire pétrifiés. Néamoins des pins \& d'autres arbres réfineus y ont pris racine \& quelques plantes peu délicates, telles qu'il en vient chés nots fur les vieux murs \& fur les rochers, prcpèrent très bien dans les fentes; ce qui prive que la terre expoiée fur des hauterrs aux vents \& à la féchereffe, quoique I: pluie enlève fes parties graffes, $\mathrm{n}^{3}$ eft cepndant pas impropre a produire des plantes. La rivière $T a$ ou $T a b s$, fe jette dans la ner au deffous de Canton. Le flux \& le sflux fait que fon eau eft riêlée ici d'eau lée. Elle partage le pais, à la diftance re quelques milles autour de la ville, en iufieurs isles grandes \& petites, dont les ivages font larges, unis \& bas, de façon que lorfque l'eau monte le plus haut, ils A 4 
reffemblent plus à des grands lacs qu'à das champs. Cette humidité continuelle doit néceffairement rendre cette terre argileufe en elle même, bourbeufe \& marécaseufe: auffi les agricoles y enfoncent-ils jufcu' aux genoux, avant que d'arriver à un terrein ferme.

Il femble qu' un terrain, qui et fous l'eau deux fois toutes les vingt quatre heures, doit relter privé de fes partie graffes, \& $\&$ de tout ce qu' il faut pour pnduire du bled, \& devient par là impropre t être cultivé, parceque l'eau emporte \& enraine lorfqu' elle s'en va, l'engrais qu' elle peut avoir amené. Ces champs humides nfemencés de ris, ne reçoivent en effet pint d'autre engraifiement, que le chaume du ris même mis dans la terre, qu' on lale pourrir: Malgré cela ils rendent tous es ans une moiffon au centuple.

Toutes les fois que l'eau inonde Is champs, elle y laiffe quelques parties gra. fes \& bourbeufes, qui rendent la terre fe. tile; car l'eau de la marée, qui monte et plus faltée \& plus trouble, qu elle ne l'el lorf fu' elle defcend: outre cela elle defcenc au commencement fort lentement \& le: champs au ris font déja à decouvert, lorfque la marée defcend avec véhémence; ce qui fait que le limon falé, qui a coulé à fond \& qui fait l'engraiflement du champ n'en peut plus être emporté. 


\section{Des champs iे ris ou rifières.}

Ts champs à ris font fi moux en quel1. ques endroits, que la marée emporte la terre des rivages. Pour éviter cet inconvénient, on y plante des cyprès; dont les racines s' entrelacent, \& donnent de la fermeté à la terre. Chaque champ de ris étant féparé de la rivière par de larges folfés, ces allées de cyprès plantées en loagues haies forment un beau coup d'oeil, particulièrement, lorfque la terre eft lous I' eau.

Dans des endroits plus élevés, qui ne peuvent pas être arrolés par la marée, on a planté une autre elpèce de champs à ris. Pour leur procurer une quantité égale d'eau, ils font une terrafte autour de chacun de ces champs de la hauteur de deux à trois pieds \& dans le temps de la pluie, ils laiffent augmenter les eaux, ou ils les lailfent écouler, felon qu'ils le jugent à propos. Dans le tems de féchereffe ils les arrofent ou par des conduits, ou en y portant l'eau. La terre de ces champs elt mêlée d'une argile \& d'une glaile ferme, \& comme ils peuvent rendre le double de ce que les autres champs rendent, on les engraifle de plufieurs efpèces de fumier \& on en a plus grand foin. Les Chinois plantent A 5 
outre cela du ris dans des endroits bourbeux \& marécagcux; mais comme il n' eft pas prffible de les entretenir dans un degré égal d' humidité fans beauccup de peine \& de dépenies, ils manquent ordinairement dans les années sèches.

Des Chinois dignes de fois m'ont racconté que dans la province rucbien, la rivière qui fe jette dans la mer près de Changebeu \& Amoy formoit des grands ri-. vages plats \& que les habitans, mécontens de ce qu' un terrain fi étendu ne produit rien, faifoient des radeaux, étendoient des nattes deffus, y mettoient de la terre, \& y plantoient du ris avec beaucoup de profit: qu'à la vérité ces champs flottans étoient quelques fois fujets à être endommagés par les ouragans, lorfque les vents changeoient ; mais qu' on les regardoit comme fort lucratifs, parce que dans le tems $f e c$, auffi bien que dans le tems pluvieux, l'eau de deffous leur confervoit toujours une humidité égale, \& que la pluie ne leur caufoit point de dommage puifqu'elle s'écouloit bientôt . C'elt là une preuve de leur indultrie digne de confidération.

On prépare tous ces champs, ou avec Ia charrue, ou avec la bêche, \& comme tout fe réduit au but que le vieux chaume de ris foit retourné \& mis dans la terre, l'une ou l'autre peut également fervir à cet 
effet; car le terrein étant toujours fi mou, comme nous avons déja obfervé, que les laboureurs y entrent jufqu' aux genoux, il eft aifé de le travailler. Leur charrue eft extrêmement fimple, \& eft tirée par un bouf: mass avec la pioche, ils peuvent auffi fans beaucoup de peine remuer aufii profondément dans la terre bourbeufe, qu'ils le jugent à propos. A la prémière marée la terre ayant été inondée devient unie, comme fi elle avoit été applatie ávec un rouleau, \& comme l'humidité continuelle empêche la terre de fe coller, ils n'ont pas befoin d'autre inftrument d'agriculture.

On traite de la même manière toutes les autres efpèces de terroir, choififfant pour cet effet le tems, où la terre eft le plus amollie par l'humidité, \& conféquemment facile à travailler. Ils engraiffent \& labourent une petite partie d'un acre, plus ou moins grande d'environ foixante pieds en quarré. Il faut à la vérité qu'elle foit humide \& molle comme le relte. il eft néceffaire cependant, qu'elle foit afsès eloignée de la rivière, pour qu' elle ne puille pas être entièrement inondée par la marée, lors qu'elle monte. Ils enfemencent cette partie fort copieufement d'un ris, qu'ils ont laiffé auparavant s'imbiber d'une eau qui a reltée fur du funier \& de la chaux. Lorique le ris commence à paroitre, ils tiennent l'acre A 6 
fous l'eau à trois pouces de profondeur; au bout de trente jours ces plantes font propres à être transplantées dans des grands champs. Lorfqu'ils les transplantent, its ne fe foucient pas beaucoup qu' elles foient en lignes directes. Ils ont foin feulement, que chaque plante ait une place fuffifante. La diftance qu' ils laiffent ordinairement entre les plantes eft de huit à neuf pouces. Ils font cette opération comme toutes les autres avec beaucoup de facilité, \& de manière, qu'ils ôtent environ la longueur de deux pouces des pointes des plantes \& mettent chacune en particulier, ou lorfqu' elle eft trop petite, plufieurs enfemble, dans la terre molle avec les doigts, afsès profondément pour qu' il y ait deux pouces de terre au deffus de racines. Quand le ris a été tranfplanté de cette manière, ils n'y font plus rien : ils examinent feulement, pendant que les plantes font encore délicates, fi elles n'ont pas été endommagées par les vers ou par d'autres infectes; \& fi cela eft arrivé, ils mettent des nouvelles plantes à la place de celles qui ont péri, \& jettent alors un peu de chaux fur le champ; ce qui chaffe les infectes. 
Des vents variables $\delta$ du climat.

Ts vents variables qui fouflent dans les L. provinces méridionales de la Chine fituées en dedans du tropique du cancer, y produifent de tels changemens dans le climat qu'ils partagent l'année en deux faifons, favoir: la faifon humide \& la faifon sèche. Quand le foleil paffe dans le mois de Septembre de la ligne équinoctiale vers le midi, l'air devient peu à peu plus frais, \& pendant le mois d'Oetobre \& une partie du mois de Novembre, il regne ordinairement des brouillards \& des petites pluies: aufif toutes les fois qu' un vent du Nord-eft s'élère, l'air s'éclaire \& refte ferein, julqu'à ce que ce vent d'hiver ait entièrement difpart. Dans les mois fuivans le climat eft pius ftable, plus fec \& plus beau, jufqu'à ce que le foleil ait achevé de nouveau fon voyage d'hiver \& ait paffé dans le mois de Mars, au travers de la ligne équinoctiale vers le Nord.

L'air échauffé, qui a tiré peu à peu en haut une quantité de vapeurs, les rend fuccelfivement par des pluies pius fortes \& plus abondantes, qui dans les mois de May \&z de Juin deviennent toujours plus copieufes \& tellement perfévérantes, qu'on voit fouvent douze à quinze jours de pluie fans dilicontinuité. De fortes tempêtes \& des ouragans 
accompagnent ordinairement les grandes fluies du Sud vers l'Oueft. Quoique le foleil commence dans le mois de Juin, à diriger fon cours de nouveau vers le midi, il laiffe cependant dans ces endroits une chaleur plus forte qu' elle n'étoit, lorfque le foleil y donnoit perpendiculairement : Le tams commence pourtant à devenir plus ttable, \& moins pluvieux, \& des nuées baffes \& des coups de vents font caufe que la chaleur, qui a refté fe fait fentir pendant quelques jours plus forte qu'auparavant. Le mois d'Aôut eft plus tempéré, mais vers le mois de Septembre le tems eft variable, tantôt lerein, tantôt nébuleux, ce qui dure jufqu' à ce que l'autre vent s'établiffe. C' eft pour cette raifon que les mois d'Avril, de May, \& de: Juin font appellés chés eux, mois de pluie ; car la pluie tombe alors plus fréquemment, $\mathcal{E}_{x}$ en fi grande quantité que l'eau fe jette en grands torrents des endroits efcarpés, \& fe forme de nouveaux canaux \& de nouveaux lits entre les rochers. Pour remédier it la féchereffe qui pourroit avoir lieu dans les mois fuivants, les habitans conduifent cette eau dans leurs champs à ris .

Il faut remarquer ici que pendant l'équinoxe, le chargement de vents eft précédé ordinairement d'une efpèce de tempête violente, qui frufle quelques jours avant ou après ia pleine lune. L'air inférieur devient 
alors extrêmement épais \& nébuleux. Ce brouillard, qui a caufe de la véhémence du vent, ne peut pas fe transformer en pluie, eft furieufement agité. L'ouragan augmente à proportion qu'il va vers l' Oueft, quand il eft de l'Oueft, les arbres \& les maifons peuvent à peine lui réfifter : il abbandonne une région après l'autre, \& au bout de vingt quatre heures il commence à fe diffiper. Les champs \& les bâtimens en font ordinairement endommagés: aufi appelle-t-on cet ouragan $T a y$, qui veut dire le grand vent.

Les Chinois favent tirer avantage, pour leur agriculture, de cet ordre fucceffif des vents: ils labourent la terre, lorfqu' elle eft mouillée par le tems de l'automne \& encore afsès molle, pour y femer, ou pour y planter, pour l'hiver. Cela fe fait ordinairement dans le mois de Décembre, \& comme l'air eft alors plus frais, l'eau ne peut pas afsès fecher, pour qu' elle ne contribue pas à l'accroiffement \& à la moilfon. Celle-ci arrive cent vingt jours après ou dans le mois d'Avril: on engraiffe alors un peu, on laboure \& on prépare ce champ que la pluie a ramolli, pour y femer ou planter de nouveau. C'elt vers la fin du mois de Miay, ou vers le commencement de Juin, qu'on prépare ordinairement les champs à ris pour la feconde moiffon de la même année. Dn leroit tenté de croire que le change. 
ment de la pluie \& de la chaleur faciliteroit l'accroiffement du ris plus promptement qu'à la prémière moiflon; cependant ils font obligés d'attendre plus longtems cette feconde moiffon \& il faut qu'ils comptent cent trente jours depuis l'enfemencement juiqu'à la récolte du ris. De là vient qu' elle n'arrive que rarement dans le mois de Septembre.

On met les plantes au ris dans les champs bas, vers la fin du mois d'Avril, ou vers le commencement du mois de May. Ces plantes demandent autant de jours pour mûrir que celles des autres champs \& la moiffon arrive ordinairement dans le mois de Septembre: après cela on laiffe la terre en friche jufqu' au mois d'Avril; pendant ce tems les chaumes 2 les racines du ris qui ont refté, pourriffent, de manière qu'ils fe confondent entièrement avec la terre quand oa la laboure.

Dès que le ris commence à blanchir, figne de fa maturité, on le coupe avec des faucilles à main, dont le tranchant eft dentelé comme une fcie; on le lie en gerbe, $\&$ on le met dans un endroit fec \& élevé, pour qu'il y leche \& qu'il y demeure jufqu'au tems qui on le bat. Le ris battu a encore fon écorce, \& on l'appelle Paddi, on s'en fert en partic pour le femer, en partie pour en nourrir le bétail: mais avant 
que les hommes s'en fervent, ils l'ecrafent dans des mortiers de pierre avec des pilons de bois, après quoi on le vanne.

Quelques aconomes, qui ont des champs trop étendus, pour qu'ils les cultivent eux mêmes, en cedent une partie à des pauvres gens, mojennant une certaine redevance. Ces fermiers font trop pauvres pour labourer les champs avec la charrue \& des boufs; c'eft pourquoi ils fe fervent de pioches, ils achetent des autres, les plantes de ris, qu'il leur faut. quand le ris eft mûr, \& qu'ils l'ont coupé, ils le battent fur quelque colline, ou quelque rocher nud, \& ils en payent la redevance au propriétaire.

\section{De l'engraiffement des terres.}

T $\mathrm{Ne}$ agriculture fi étendue exigeant beaucoup d'engrais, les pauvres gens gagnent leur vie à ramaffer dans les rues \& aux environs des naifons \& même avec des petites fampanes ou barques fur les rivages, toutcs fortes de matières propres à engraiffer, même les excrémens d' hommes $\&$ de bêtes; ils les vendent à ceux qui en font un commerce particulier. Ceux-ci les revendent aux agricoles qui en ont befoin . Ils amaffent auffi l'urine dans des vafes particuliers, qu'ils tiennent dans les maitons. Quand la moilion a été avautageule, 


\section{8}

un Peleul (a) de la prémière efpèce d' engrais coute deux Mes (b), \& un Pekul de la dernière ne coute que la moitié : outre cela chaque oeconome a foin que les excrémens du bétail ne foient pas perdus dans les patis on emploie des enfans ou d'autres gens qui ne font pas en état de s'occuper plus utilement, à les ramaffer. Ils brulent auffi les offemens qu'ils trouvent, \& en jettent la cendre avec celle d'herbe \& de bois brulé fur les champs, pour les rendre plus fertiles.

On engraiffe, on laboure \& on applanit les champs qui quoiqu' humides, font dans une fituation plus élevée que ceux dont nous avons parlé jufqu'ici. Leur terre eft plus meublée: on enlemence fort copieufement une portion d'un champ, d'un blé, qui a été trempé pendant quelques jours dans une fauce de fumier \& on le tranfplante. Quelques fois on plante auffi le blé trempé de cette manière dans le champ préparé, de façon que les grains font à la diftance de quatre pouces l'un de l'autre. on preffe la terre autour de chaque grain. Dans une grande féchereffe, on conduit une petite quantité d'eau fur les champs. Les

(a) Un Pekul peée enviroa cent quarante deux livres \& demi poids de Suède.

(b) Un Mes, qui eft la dixióme partie d'un Tell, vaut dix fols de France. 
profonds fillons, qui fe font formés de la preffion de la terre contre les grains, reçoivent alors l'eau \& donnent de l'humidité aux jeunes plantes fans les noyer. La véritable faifon pour tranfplanter eft vers la fin de Décembre, quoique l'air foit alors fort frais \& qu' il fafle froid quelque fois pendant la nuit. La femence pouffe pourtant, $\&$ forme fa fouche au bout de quinze jours, dont chacune donne dans le mois de Mars fept à neuf tiges avec leurs épis, mais la paille eft plus cotirte que chés nous. Le mois de May donne une moiffon abondante: On m'a affuré qu'un grain du bled, en donnoit cent vingt, ce qui recompenfe bien le travail qu' on y a mis.

Comme le ris eft la principale nourriture des Chinois, puifqu' ils s'en fervent en guife de pain, on n'emploie qu' une petite portion du terrain pour le blé: Ils s'en fervent uniquement pour leurs conftures, dont ils confomment une grande guantie ta leurs jours de fêres, pour leurs Pagodes \& leurs offrandes. Ils en font aufi un peu four eux mêmes. Les étrangers en confomment la plus grande quantité; \& comme le blé, que cette province produit, n'eft pas fuffifant pour eux, on en apporte en quantité des provinces feptentrionales pour leur ufage.

J'ai vu dans un petit clamp, de l'orge dans le mois de Juin, qui avoit très bien 


\section{DE L'ECONOMIE RURALE}

pouffé : mais comme on l'avoit femé trop tard, la chaleur, qui étoit déja brulante, l'avoit fait monter fi vite, que la tige fe flêtrit, avant qu' elle pût former des grains, $\&$ qu' elle ne contenoit dans fes épis confiderables que des ecoffes vuides. Si on avoit ¿emé cet orge, comme l'on fème le blé dans un tems plus frais, il auroit fourni rans doute une riche moilfon. J'en concluois que comme ces elpèces de blé profpèrent , très bien étant femées \& tranfplantées dans un champ bien préparé \& également humide, le tems frais eft plus convenable à leur accroiffement que le tems chaud.

Leur manière de battre le bied \& le ris eft la mème \& fe fait comme chés nous avec des fléaux. Après que le bled elt battu, on le fait paffer par une macchine faite exprès pour le nettoyer, \& qui en fait partir toute la pouffiere, avant que de le moudre. Si les moulins à Canton étoient auffi commodes, que ces machines, pour netoyer, ils pourroient épargins: bsaucoup de travail \& de mains; car la manière de moudre ici avec des moulins à main eft extrêmement penible. Il edt étrange, que les Chinois, qui ont tant d'inventions ingénieufes pour faciliter des petits travaux, faffent tout avec lcurs mains dans les grands travaux, comme fier, moudre \& d'autres qui exigent plus de force, quoiqu'ils ayent 
afsès de facilité pour conftruire des machines tant fur les montagnes, que fur les rivières.

Ainfi, comme nous venons de voir, ils emp'oyent à l'agriculture tous les endroits plats \& bas \& mettent peu de travail à la terre molle qu'ils tiennent entièrement ésale. La récolte rend ordinairement au centuple: mais loriqu'il furvient un tems déréglé, une trop grande féchereffe ou une trop grande humidité, il y a de la ftérilité ici comme ailleurs: \& comme le païs eft prodigieufement peuplé, elle a toujours des grandes conféquences: une petite augmentation du prix du ris fait murmurer les pauvres \& les fainéans, \& quand enfin d'autres s'attroupent avec ces mécontens, il en naît une révolte contre le gouvernement tartare, ce qui arriva l'an. $175 \mathrm{I}$., la famine étant accompagnée encore d'une maladie epidémique, qui enleva beaucoup de monde.

Des champs fitués fur des hauteurs.

Es hauteurs \& des pentes feroient impropres par leur fituation à porter quelque chofe. Dans les mois pluvieux : la pluie fréquente noyeroit \& emporteroit tout ce qu'on auroit femé, ou bien les plantes, après que l'eau s'elt écoulée, fe trouveroient dépourvues de terre \& expofées ainfi à la 
féchereffe \& à la chaleur qui furviendroit. Pour remédier à cet inconvénient, ils ont eu foin de changer les hauteurs en plaines, moyennant des terraffes dont la hauteur \& la largeur eft fuivant la pente. Ils employent ces terraffes à différentes plantes, \& donnent à chacune la place qui convient le mieux à fa nature. Celles qui fapportent le plus de féchereffe, ont leur place en haut, \& les plantes qui font plus tendres, font placées en bas. Quand la pluie à mouillé la terre des terraffes fupérieures, on conduit l'eau aux terraffes inférieures, par le moyen de fillons: ainfi outre la pluie qu'elles ont reģùe, elles profitent encore de l'eau fuperfue des terraffes fupérieures.

Les bords des terraffes qui font faites quatre ou cinq pieds l'une au deffus de l'autre, deviennent quelques fois fi durs par l'effet de la pluie \& du foleil, qu'ils pourroient fubfitter nombre d'années: malgré cela ils y ont planté plufieurs arbres, dont les racines entrelacées donnent de la confiltance à ces bords. Les arbres mêmes garantiffent les plantes, de la chaleur du foleil \& des vents, $\&$ font que les terraffes ainfi ornées offrent à la vue ur fort bel afoect.

Quand ils ont remué la terre des terraffes avec une petite charrue, ou avec une bêche, \& qu'ils l'ont applani avec un râteau au tien d'une herfe, on lui donne qualques 
fois, pendant qu'on la laboure autant d'engraiffement, que les plantes qu' on veut y mettre en exigent; mais en cela aufi on oblerve une grande économie : on trempe pour la plupart le fumier dans des trous ronds murés dans la terre \& remplis d'eau, on arrofe la lémence avec cette fauce, quelques fois en plantant ils mettent une poignée de cendre fur chaque grain, ils croyent, que l'engraiffement, qui tombe entre les plantes n'eft d'aucune utilité.

On laiffe à peine un mois de repos aux couches conftruites fur les terraffes, ou autrepart, \& dès qu'une production eft mûre \& recueillie, on les "répare pour en porter une autre, ce qui fe fait trois fois par an. Quant à la faifon, les cultivateurs font attention à la nature des végétaux, \& l'on donne à chaque plante, la faifon la plus convenable, felon qu' elle demande ou de l'humidité ou du froid ou du fec. Les racines feules ont pour partage l'automne.

Les genres de fémences, qu'on plantoit le plus généralement fur ces hauteurs, étoient les fuivans: un genre de fémence groffière d'une plante avec une racine mince qui reffemble pour les feuilles, les Heurs \& les vaiffeaux qui confervent la graine à nos radis. Le commencement de Décembre étoit le tems le plus convenable pour celle là on formoit dans la terre nouvellement labourée 


\section{De L'Economie RURALE}

des fillons qui avoient un pied de large, \& 8 un demi pied de profondeur. Entre ces fillons il y avoit des longues couches étroites d'un quart d'aune de largeur : au moyen de ces fillons l' eau trop abondante pouvoit découler, après avoir laiffé une humidité fuffilate. on plantoit les grains à la profondeur de quatre doigts \& on leur laiffoit fept à huit pouces d'intervalle, comme cela fe fait dans la faifon fèche, on les arrofe au commencement . au mois de fevrier tout étoit en Heurs \& dans le mois d'avril les vaiffeaux à femence devenoient jaunes; alors on arrachoit les plantes, on les léchoit $\&$ on les battoit pour en avoir la femence abondante. De cette fémence on tire une huile, dont on fe fert beaucoup dans le ménage particulièrement pour les lampes, \& quand elle eft fraiche, on s'en fert pour préparer les. mets. Cette huile eft $f i$ graffe, qu' on ne peut pas l'employer dans la peinture, parcequ' elle ne fèche pas alsès. Le noir de fumée qui fort de ces lampes fait la couleur noire, connue fous le nom d'encre de la Chine.

Ordinairement la lémence de Coton, qu'ils appellent Minfu, prend la place de la lémence à l'huile. On prepare le terrain, comme on a déja decrit, on plante auffi la fémence dans des couches, aufi étroites, que pour la fémence à l'huile à la diftance d'un pied l'une de l'autre. Il faut remarquer, que felon 
Ion que les plantes font plus fortes ou s'etendent davantage, ils font les couches plus larges ou plus étroites, plus eloignées ou plus rapprochées l'une de l'autre. C'eft dans le mois d'Auril, qu' ils mettent les grains dans la terre. Ils jettent fur chaque grain quelques poignées pleines de cendre de l'herbe, qui produit l'huile, ou d'une autre; \& c'eft là tout l'engrais qu'ils donnent pour cette fois à la terre. Jufqu'à ce que la quatrième feuille pouffe, on l'arrofe dans des jours fecs. La chaleur \& la pluie font que les fleurs qui ont paru dans le mois de Juillet, fe changent dans celui d'Aôut en fruits, qui mûriffent dans un tems fec \& s'ouvrent pour montrer le coton .

Alors on les cueille, on fépare le coton $\&$ la fémence, qu' on garde pour l'enfemencement prochain. Trop d'humidité nuit à la plante du coton, pendant le tems de l'accroiffement \& pendant celui de la maturité: auffi quand le tems pluvieux continue, le coton pourrit fur la tige; ce qui fait que la récolte n'elt que médiocre, en comparaifon des autres. Les fouris recherchent extrêmement cette fémence, non feulement lorfqu' clle $\in$ ft étendue après qu'on la cueillie, mais même quand elle mûrit encore dans fes vaiffeaux .

Les patates qu' ils appellent Fauciy, font le troifieme $\&$ dernier fruit, qu' ils plantent B 
fur les terraffes : après le coton, ils remuent de nouveau la terre, \& y mettent des petits morceaux des patates coupés à la diftance d'environ un pied. Comme ce fruit n'eft pas aulfi délicat, que le précédent, qu'il croît lentement, Sx qu'il réfifte au froid, ils lui laiffent pour croître les derniers mois de l'année. Ces patates different des nôtres en quelque chofe. Elles ont la pelure rouge, elles font plus longues, jaunes \& d'un goût doux \& agréable; mais l'herbe reffemble à celle de nos patates en Europe.

Ils ne font pas toujours fuccéder le coton à la plante, à l'huile, \& les patates au coton. D'autres végétaux co mme des tentilles, des fèves, des Locktau \& des Calebaffes prennent quelques fois la place du coton; mais ordinairement ils commencent par la fémence à l'huile, \& ils finiffent de tirer parti de leurs terraffes, pour l'année, par des patates. Ils préparent toujours le terrein de la manière décrite \& ils ne mettent point de fémence, qui n'ait été trempée auparavant pendant quelques jours dans de la fauce de funier, ou dans de l'eau de chaux.

Ils plantent \& traitent comme les patates, les Yams, qu'ils appellent Utau: mais le terrain pour les rams eft différent; car on plante ces racines dans des endroits fi marécageux \& fi humides, qu'ils ne feroient 
pas propres pour d'autres plantes, quelque fois aulfi dans un champ de ris, qui a déja fervi une fois dans une année, \& qu' on n'eltime pas afsès bon, pour fournir une feconde récolte. Plus on laiffe ces racines en terre, plus elles deviennent grandes ordinairement on les tire de la terre dans le mois de Novembre.

Ils mettoient les racines coupées de la canne de fucre, dont chaque morceau avoit deux jets, dains la terre, à la profondeur de plus d'un quart d'aune, \& ils laiffoient deux pieds d'efpace entre chaque rang. Ils employent four cela auffi bien les terraffes les plus élevées, que les endroits les plus ba: Dans les mois de Mars \& d'Avril, on la planta dans des endroits bas, \& dans les mois pluvieux fur des hauteurs; ce qui produifit une récolte différente. Lorfque la canne commençoit à jaunir, on la coupe; car fi on laiffe plus long-tems, elle commence à pourrir par la racine. Elle atteignoit la hauteur de quatre à fix aunes. Ils portent à un endroit commode \& fitué fur la rivière, quelques charges d'une fampane de Sucre, y conttruifent une maifon de Bambou \& de nattes. à l'un des bouts de cette maifon ils font . un four avec deux grands chauderons, qui y font mûrés; à l'autre bout, il y a une aire fpacieufe garnie de planches, fur laquelle deux boufs trainent un roulsau équarri fait B 2 


\section{DE L'ECONOMIE RURALE}

d'un bois dur. La canne, qu' on avoit mis fous le rouleau par couches, étoit ecrafée de cette manière, \& le jus qu' on conduifoit, moyennant un égout au bout de l'aire, s'y raffernbloit dans un grand vaiffeau. On mettoit la canne ainfi preffée dans le chau. deron bouillant pour en tirer tout le fuc. On le mêloit enfuite avec ce qui étoit déja exprimé, on le failoit paffer par le crible $\&$ on le laiffoit bouillir dans l'autre chauderon, jufqu'à ce qu'il prît la confiftence d'un fucre brun .

Les feuilles \& les cannes, qui ne contenoient plus de parties fucreules, fervoient pour fournir le feu néceffaire. Quand il n'y avoit plus de provifion de canne dans un endroit, ils défaifoient dans la maifon, \& emportoient les uftenciles. Ces rafineurs de fucre parcouroient le paîs \& tiroient le fuc de la canne des agricotes. D'autres rafineurs l'épuroient après \& en faifoient de la caftonade plus ou moins fine.

\section{Des jardins potagers.}

Comme à l'exception de quelques jardins imparfaits de ce genre, je n'ai pas eu occafion d'en voir, la defcription que j'en vais faire, ne fera pas autri complette que je le voudrois. Ce que j'en puis dire, eft qu'ils choiffent pour ces jardins ordinaire- 
ment des endroits bas \& argilleux \& qu'ils n'y épargnent pas l'engrais. Les plantes comnues étoient de la Salade, des longs \& des courts concombres, du Purjo, des oignons blancs, du célery, des épinards, des radis longs, des carottes, de l'arroche rouge, une efpèce de raves aqueufes, des melons d'eau \& d'autres : Ils en ont reçû originairement la fémence des Portugais. On y trouve encore une grande variété de plantes, dont le nom \& la figure nous eft entièrement inconnu. Le Pourpier croilfoit fauvage; mais ils ne s'ea fervoient point \& par conféquent n'en faifoient point de cas. Ils avoient une efpèce groffière d'epinards aqueux dans des étangs, qui avoient une demi toife de profondeur. Il y étoir fi abondant, qu'il en couvroit la furface, ils en font un grand ufage dans leurs cuifines. Ils plantent le gingembre par petits morceaux dans une terre graffe \& argilleufe à la profondeur de quatre doigts; ce qui fe fait aux mois de Février \& de Mars. Plus tard, la chaleur poufferoit trop la tige \& les feuilles, la racine deviendroit fpongieufe \& demeureroit petite. Au refte cette plante fupporte le froid \& le chaud.

Le Tabac chés eux s'appelle rien. I culture de cette plante leur eft d'autant plus avantageufe, qu'ils en font cas à la Chine plus qu' en aucun lieu du monde. 
Ils n'y épargnent ni foins ni bons terrains. On le plante le mois de mars, chaque plante à la diftance d'un pied \& demi l'une de l'autre. Le mois d'Aôut on le cueille, on le laiffe reffuer $\&$ enfuite on le travaille comme chés nous. Ce Tabac ne paroit pas être de la meilleure qualité : il reffemble fort au nôtre; mais l'odeur \& le gout en font médiocres. Les Chinois lui donnent la préférence fur celui de Manille \& d'Aynam, Bréfil. Les feuilles defféchées \& miles en preffe les unes fur les autres, font coupées par bouts avec un inftrument de fer dans la même forme, qui elt ufitée chés nous, quand on le fume. Il diftille une huile gluante \& d'une odeur forte. Coupé par morceaux plus gros, il fume mieux. Le débit de cette marchandife eft fi confiderable, qu' on en apporte ici une grande guantité de toutes les contrées voifines.

Ils difporent per rangs fur de larges couches une plante, qui reffemble à la menthe, mais dont les feuilles font plus pâles. Ils l'appellent Fockiyong. Le mois de Mars, elle a un pied de haut. Sa culture demande beaucoup de foins, on la feme dans le tems froid; dans le tems de la chaleur, pour en prévenir les inconvéniens, on la couvire \& $z$ on l' entoure de nattes Iis eitiment beaucoup cette plante. La mefure d'un Pekel s'en vend cinquante Tell ( $\left.{ }^{*}\right)$ on

(*) Un Tell vaut cent fols de Fraace. 
la croit très bonne contre la confomption .

L'arbre merveilleux (Ricinus) de la grande $S x$ de la petite efpèce à été apporté ici d'Aynam. Ils en mettent partout \& fans ordre dans leurs jardins \& particulièrement das petits. Le fruit fous le preffoir, rend une huile blanche \& claire en grande quantité. Après en avoir óté la graiffe par le moyen du minium, de la chaux vive $\&$ de la terre vitriolique, ils en font un vernis, qui fert à la peinture. Ce vernis fèche promptement $\&$ donne un eclat fort vif.

Ils font ufage au lieu du chou, d'une plante, qui reffemble au glouteron par fes feuilles grandes \& groffes, dont les pédicu= les épais fortent d'une racine mince. La fleur eft jaune, la tige contenant la graine \& la graine même reffemblent à celles du chou. Comme ils l'employent journellement, la confommation en elt grande. Cette plante croît fort vite \& dans toutes les laifons dès que la récolte en eft faite, ils en relsèment de nouvelle fur la même couche, on la cuit à demi \& on la laiffe fécher. C'eft une de leurs provifions dans leurs voyages lur mer. Les Tartares ont apporté ici, de Pekin, une efpèce de chou blanc qui a la tête longue \& étroite, il n' eit pas encore fort en ulage; ainfi il n'eit pas commun. 


\section{Des Arbres.}

Uoiqu'il y ait ici plufreurs efpèces de bons arbres fruitiers, on ne remarque pas que les Chinois s'appliquent particulièrement à cette culture. Parmi la grande diverfité $d$ 'arbres, qui ornent leurs jardins ix leurs terraffes, il s'en trouve de cette dernière forte. Ils ont même de grands jardins tout plantés d'arbres; ce qu'ils regardent comme une grande magnificence. C'elt pourquoi les environs de leurs pagodes $\&$ de leurs maifons de plaifance en font deccrés; mais la pluparc de ces arbres nous font inconnus.

L'oranger, que les Portugais ont tranfplanté en Europe, porte ici de gros \& bons fruits. On dit que dans le canton de Fockien $\&$ dans les environs d'amay, ils font encore plus parfaits. Il y en a ici de differentes fortes: quelques uns font de la groffeur des noix de Galle; d'autes font comme des reinettes. Il y en a qui ont la forme angulaire \& la couleur rougeâtre Sxc. Il eft rare qu' on prenne ici un loin bien particulier de la culture de ces arbres, \& qu' on leur donne même une certaine difpofition \& quelque arrangement . Lorfqu'ils fe trouvent placés à l'abri des vents violens, ils viennent fort bien d'eux mêmes \& rapportent en abon. dance. Les provinces de Fockiers \& de Quan- 
tung font obligces d'envoyer tous les ans une grande quantité de fruits à la cour de l'eling.

Le Leichi eft un arbre que les Chinois paroilfent eltimer autant que l'oranzer. II y en a de différentes efpèces, de gros, de petits, \& de fauvages: les fruits font de la groffeur d'une noix mufcade. Ils font enrourés d'une écorce rude, raboteufe \& rougeâtre, ils croiflent comme la vigne en forme de grappe. L'arbre atteint la hauteur du poirier \& eft garni de feuilles petites pointues \& piquantes. Les fruits fe confervent déffechés \& ont le gout des raifins de Corinthe. Il femble preiqu' incroyable que les environs de Canton, qui elt le feul païs, où cet arbre vienne, produife par année pour cent mille Tell de fruits de Leichi déffechés.

Le thé, qu'ils appellent Chia, \& qui croît ici dans une isle vis à vis de Cantor elt en réputation pour fa vertu contre les maladies de poitrine.

L'isle s' appelle Honam, \& fon the Thée d' honam. L'arbulte qui elt de la hauteur d'une aune ou d'une aune \& demi, s'elève par rangs fur des collines fèches \& fabioneufes. On ceuille les feuilles tendres \& qui font d' un verd clair, le mois de Mars, on les rôtit dans des chauderons de fer, \& $x$ on les difpole en forme de rouleau comme les autres efpèces de 'The, on néglige les feuilles dures \& qui 
34 De l'Economie ruRALE

font d'un verd plus foncé. Il paroit, qu'on ne prend pas grande peine pour la culture de cet arbre; on en laiffe dépérir plus de la moitié.

L'arbre d'Areca ne croît pas loin de Canton, ainfi que je le conjecture par des noix fraiches, que j'en ai vues ici. Il y avoit dans l'isle d' Aynam diverfes plantations de cet arbre. Le terrain, qui le porte eft gras \& humide. L'arbre reffemble au cocotier, \& a la tige droite. Lorfque le fruit eft mûr, l'écorce prend une couleur jaune. La noix diffère peu de la noix mufcade. Elle fe fèche $\&$ s' envoie dans les provinces feptentrionales.

La plante du Béthel n'eft point délicate. Elle croit d'elle même fans culture, lorfqu' elle trouve un terrain propre. Ses feuilles frottées avec de la chaux \& la noix d'Areca, font le Pinang fi connu que ces peuples \& ceux des autres païs orientaux mâchent avec tant d'appétit.

Le Manglier s'elève fort haut \& porte des branches fort étenduës comme le frêne. La feuille eft du genre de celle de l'aube epine \& le fruit paffe pour un des plus falubres des Indes.

Le Pumpelmose eft une efpèce de gros citron doux, l'arbre reffemble au citronier; mais les feuilles font plus larges.

Ils ont de petits citrons aigres, des Longans \& plufieurs autres fortes de fruits. Ils 
ont aufi de l'Otornchou, dont comm: nous l'apprend le Comte, ils confervent la gomme pour leur vernis.

Il feroit trop long de faire le détail \& la defcription de leurs différentes efpèces d'oliviers, de poiriers, de pommiers \& de raifins. On ne peut-pas dire qu'ils donnent quelque préférence à une culture fur une autre: ils les laiffent prefque tous fauvages. Ils font unage du grêffe pour quelqu'elpèce d'arores, $\&$ ils fe lervent de cette méthode fort heureufement .

\section{Des Jardins de plaifance.}

Omme le gout des Chinois diffère beaucoup de celui des autres nations pour les manières, l'habillement $\&$ c. cette différence n'elt pas moins remarquable dans leurs jardins de fleurs \& de pur agrément. Ils le foucient fort peu de parterres, de haies, d'allées couvertes \& généralement de la fymmetrie: une place nue, ornée de pierres de différente grandeur \& couleurs, qui forment des figures de dragons \& de fleurs, leur plait beaucoup plus, qu' un parterre orné de beaux deffeins, dont les interltices font remplis d'herbe. Leurs allées ne font pas ouvertes, elles ont pour la plâpart des murs au"- ôtés, contre lefquels on a planté des vignes cu d'autres arbres qui graviffent le long des murs.

B 6 
Ils les tirent moyennant des bâtons d'un mur à l'autre \& couvrent ainfi l'allée. Les hancs font pratiqués dans des allées, qui n'ont point de murs aux côtés \& par l'arrangement des pierres, ils forment de nombreuies cavités, où ils placent des vafes de différentes tleurs. Les allées font difpofées en courbures; quelquefois elles fe prolongent au delà d'une petite place unie, garnie de pierres, \& menent à une maifon de plaifance découverte, fur laquelle il $\mathrm{y}$ a des vafes de fleurs . Quelquefois elles paffent par des arcades formées, avec des Bambou $\left({ }^{*}\right)$ minces qui font doubles \& arrangés inégalement. Les intervalles font remplis d' une efpèce de pervenche qui les traverle, \& qui les fait afsès reffembler à un paroy, qui a un grand trou; avec cela l'on trouve bien de la variété : des montagnes couvertes de broffailles, au pied defquelles coulent des ruiffeaux. Ces montagnes reprélentent des déferts \& font entourées d'arbres touffus \& ferres. Des bâtimens de trois à quatre étages, qui pour la piûpart font ouverts d'un côté, des tours, des grottes, creufées obliquement, des ponts, des étangs, des en. droits femés de haricots, des bocages arrangés dans le gout fauvage, des petits bois de

(*) Bambca efpica d' arbre. 
plaifance, \& d' autres variations, qui forment une belle perfpective. Ils ont auri des tables de pierre, à l'ombre de hauts arbres, ou dans des entroits élevés, dont la vue s'étend au loin.

\section{De leur bétail.}

A Ux environs de Canton \& dans les A provinces lituées fur la mer, les habitans s'appliquent fort peu au gros bétail, parcequ'il n' eft pas auffi néceffaire ici, qu'il l'eft dans les provinces contigues \& feptentrionales. Car ils peuvent labourer leurs champs fans beaucoup de travail \& fans le fecours des bêtes, \& leurs voyages \& $\operatorname{tranf}$ ports fe tont par eau; ce qui leur eft facilité par la marée. Le bouf n' elt pas un mêt agreable pour eux \& il eft remplacé par les poiflons, qui y abondent. A l'exception des Mandarins \& des officiers de guerre, il n'y a que peu de perfonnes, qui aïent des chevaux. Ils ne fe fervent pour l'agriculture que de bœufs \& de bufles; ce qui a particulierement lieu dans les endroits éloignés de la rivière: \& $z$ ce n'eft que pour en conferver la race, qu'ils nourriffent quelques vaches, parce qu'ils ne fe lervent que rarement du lait. Autrefois ils faifoient encore moins, de cas de betes à corne. Ils ae fe font portés à élever plus de boufs $\&$ 
de vaches, que depuis que les Européens ont frequenté le pais davantage, \& qu'ils en confomment annuellement une bonne partie, tant pendant leur féjour, que pour leurs provifions, lorfqu'ils s' en retournent.

Les moutons ne font pas fi communs aux environs de Canton, que dans les provinces adjacentes. On fe lert de leurs peaux \& de leur laine pour l'habillement dans les mois froids; mais elles font affès chères; auffi n'elt ce pas l'affaire de tout le monde de nourrir du bétail particulièrement des moutons.

On n'a pas autant d'ânes aux environs de Canton, qu'on en a plus loin dans le pais, où l'on fe fert d'eux pour le travail \& pour voyager. Les $T$ artares trouvent tant de gout à la chair de cet animal, qu'ils ont introduit la mode de les tuer comme les chevaux \& d'en manger. J'ai vu vendre de la viande d'âne ici .

Mais s'ils négligent d'avoir grand foin du bétail, dont nous venons de parler, ils font d'autant plus de cas des petites bêtes qu'ils nourriflent avec moins de peine \& dont ils tirent plus de profit. Ils ont tant d'expérience \& d'adreffe pour cette partie économique, qu'elle fournit une fubfiftance abondante à grand nombre de petites familles.

Comme ils mangent journellement du cochon, en quantité \& avec grand appétit, ils en nourrilfent un grand nombre. La race 
des cochons eft fertile ici \& vient bien. Les truyes donnent des cochonnets, avant que d'avoir atteint un an. Au commencement elles n'en donnent pas tant qu'à la troifiéme ou quatrième portée, qui lont ordinairement de dix fept ou dix huit cochonnets \& il en périt rarement. Les diftillateurs du Samfu (a) ceux qui pilent le ris, \& ceux qui out des moulins, nourriffent toujours beaucoup de cochons. Les pêcheurs \& ceux qui demeurent fur le rivage, en ont un nombre plus confiderable encore, parceque le poiffon dont ils les nourriffent, ne leur coute rien; ce qui leur donne cependant un gout huileux. Outre cela prefque toutes les petites familles, qui demeurent fur de Sampanes (b) nourriflent des cochons, tant pour leur propre ufage, que pour en vendre. Lorfqu' on voit combien de cochons tant crus que rôtis, ils portent par les rues, pour les vendre, \& combien ils en confomment journellement, le lard coupé par morceaux faifant pour l'ordinaire leur principal plat; qu'outre cela il leur faut de grands cochons, qu'ils rôtiffent

(a) Efpèce d'eau de vie diftillée de ris .

(b) Sampane: c'eft ainfi, qu'on appelle les barques chinoifes fans quille, qui ont prefque la forme d'un auge. Il y en a de différente grandeur, \& de différentes efpèces. Elles font ordinairement couvertes \& habitées. 
tout entiers pour leurs jours de fète, î qu'ils en facrifient auff beaucoup dans les Pagodes, on eft étcuné, qu'il puiffe y en avoir un nombre fi prodigieux; d'autant plus, qu'iis en employent beaucoup dans Icurs voyages de mer, \& qu'ils en vendent aufii aux Européens. Les cochons de lait qui proviennent de la prémière $\&$ de la feconde ventréc de la truye reftent petits, de méme que les truyes qui donnent des cochonnets, trop tôt. De là vient, qu'on châtre les cochons de lait deltinés à êtres tıés.

Ils nourriffent beaucoup de poules, plus cependant pour les étrangers, que pour eux inêmes. Ils favent chaponner avec beaucoup de dextérité. Ils font éclore les petits potilets par les poules, \& ils ne fe fervent point de fourneaux pour cela. Le climat chaud $\&$ le grand nombre d'oufs que font les poules, contribue beaucoup à leur propagation.

Il y a des faifans aux environs de Canion, mais ils n'y font pas fi communs, que plus avant dans, le païs, où on les trouve beaux \& de différentes couleurs: auffi les porte-t-on à Canton comme de raretés, \& ils coutent cher.

II n'y a point de cocqs d'Inde dans la Chine, \& quoique les vaiffeaux en apportent quelques uns tous les ans de la côte de Malabar \& de Coromandel, qui en eft la véritable patrie, les Chinois n'ont pas 
eflayé, d'en introduire l'efpèce chés eux.

Des pigeons de différentes efpèces profpè-1 rent \& fe multiplient très bien ici, de même que les oyes. Celles ci font plus petites, que les nôtres, \& reffemblent à nos oyes fauvages, pendant que leurs oyes fauvages ont de la reffemblance avec nos oyes domeftiques.

Ils entendent parfaitement bien l'éducation des canards. C'eft après les cochons, ce à quoi ils s'attachent le plus; \& comme les canards font le plat prefque ordinaire des gens qui font à leur aife, la grande confommation, qu' on en fait, exige qu' on s'applique à faciliter la propagation de leur efpice. Le climat qui eft conftament doux \& le voifinage de la rivière y contribuent beaucoup, par la commodité de les nourrier de petits poiffons \& écreviffes qui rettent fur les champs à ris, après que l'eau s'eft ecculeé, \& par conféquent à fort bon marché. Bien des Cantonois ne vivent que du commerce des canards. Les uns achecent les œufs, \& en font trafic, d'autres les font éclore dans des fourneaux, $\&$ d'autres encore élèvent les petits canards. Les fourneaux pour les couver font extrêmement fimples. On pofe une plaque de fer fur un foyer muré, on met fur la plaque une caiffe de la hauteur d'un demi pied, remplie de fable, ou on a mis les oufs en rangs. 


\section{DE I'ECONOMIE RURALE}

On les couvre d'un tamis, au deffus du quel on met une natte. Pour les échauffer, ils fe fervent de la braife d'un certain bois, qui brule lentement $\& x$ entretient une chaleur égale. D'abord on ne leur donne que peu de chaleur, peu à peu on l'augmente, jufqu'à ce qu' elle devienne afsès forte, pour faire éclore les œufs. Si quelque fois ils augmentent trop la chaleur, les jeunes canards fortent trop tôt, \& meurent ordinairement au bout de trois ou quatre jours. On vend les jeunes canards éclos de cette manière, à ceux qui les élèvent. Ceux-ci éprouvent de la manière fuivante, s'ils font éclos trop tôt: Ils prennent les jeunes canards par le bec, laiffant le corps fufpendu. $S^{\prime}$ ils s'en défendent, battant des pieds \& des ailes ils font bien \& duement éclos; mais quand ils ont reçu trop de chaleur, ils rettent tranquilles pendant qu' on les tient par le bec. Quelquefois ces derniers demeurent vivans, juiqu' à ce qu' on lailfe aller tous les jeunes canards à l'eau; ce qui arrive ordinairement environ huit jours, après qu'ils font éclos. Alors ils vacillent, fe jettent fur le dos \& meurent après quelques convulfions. On les tire cependant de l'eau \& on les laiffe fécher, puis qu' ils reviennent quelque fois; mais lorlqu' ils font mouillés de nouveau, ils meurent fort fouvent d'un parcil vertige. Quand l'eau s'eft 
¿coulée, on ramaffe les petites écreviffes, $\&$ les crabes, on les fait bouillir \& on les hâche; \& au commencement on ne nourrit les jeunes canards que de cette pâture. Quelques jours après, on y mêle du ris bouilli \& des herbes hâcheés. Quand ils font plus âgés, on les porte dans une grande Sampane dont le plancher fait de bois de Bambou, s'elève au deffus du niveau de l'eau. Elle eft entourée d'une galerie \& d'un pont qui s'abaiffe vers l'eau, on donne aux jeunes canards une vieille marâtre qui les mêne, lorfqu' on les laiffe defcendre le pont four aller paître. La vieille canne eft tellement accoutumée au cris qui vient de la Sampane, lorfqu' on veut les raffembler le foir, qu' elle y arrive, moitié en rageant \& moitié en volant. Ils changent alors de place avec leur fampane, \& abordent à un endroit, où il y a plus de nourriture pour leurs canards, \& ils les laifient aller journellement au rivage fur les champs à ris. On eft etonné de voir ces fampanes entourées de milliers de canards grands \& petits. Et ce qu'il y a de fingulier, c'eft que quand plufieurs Sampanes laiffent paître leurs canards au même endroit \& qu'on les appelle le foir, chaque canard fait retrouver la fienne. Les Chinois s'occupent conftament de la propagation des canards, exceptć lfs trois mois d'hiver; \& quoiqu' e!le exige 
beaucoup de foin, on ne voit pas que ce foin les fatigue beaucoup; car dès que les jeunes canards ont atteint l'âge de quinze jours, ils font en état, de pourvoir à leur nourriture eux mêmes.

- Les vers à foye mériteroient par leur utilité, qu'on en parlât ici, ainfi que de la manière dant on les traite; mais comme on en trouve des dètails dans d'autres rélations, je les paffe fous filence, \& je me contente de remarquer, que les Chinois mangent ces vers avec beaucoup d'appétit, après qu'ils en ont devidé la foie. Ils les font pouillier frais, ou ils les sèchent un Catti (a) de ces vers fechés vaut huit à neuf Candarins (b).

On prétend, que vers Clingebiau il y a une efpèce de vers à foye fort gros, dont on tire une foie fi épaiffe, qu' elle reffemble d'abord à du chanvre. Les habitans en font cependant une efpèce d'étoife qui, quand elle eft neuve a l'air d'une toile crue, mais par l'ufage \& par un blanchiffage répété, elle obtient du lufte, \& fait un meilleur effet. II femble que cette foie ne fe laiffe par teindre, puis qu' on ne lui donne jamais cet apprêt. On affure qu' elle

(a) Un Catti fait une lirre \& un quart ds nôtre poids.

(b) Petite monnoie. Chinois. 
eft d'une durée incroyable. On l'appelle Chinchiau, de l'endroit d'où elle vient.

\section{De la pêcbe.}

1 Ans ce païs dont la côte abonde en une grande variété de poiffons, le Taho, rivière longue \& large à fon embouchure, paffe pour être la plus fertile en toute forte de poiffons. On feroit tenté de croire que le flux \& le reflux empêcheroit la pêche, particuliérement dans les endroits efcarpés \& incommodes pour le filet; cependant ils prennent une grande quantité de poiffon de cette manière là. Voici leur manière la plus ordinaire de pêcher: Ils mettent des longs bâtons ou paliffades dans les bancs de fable éloignés du rivage, à la diftance d'une toife l'un de l'autre. D' un bâton à l'autre, ils attachent des naffes teintes en noir \& tricotées d'un fil fort. Cela fait que les poiffons, qui vont le long du rivage, s'y prennent. Cette pêche reflemble à nos naffes que nous mettons, dans les rivieres.

Ils ont auffi une quantité de paniers, qui font faits de ferches de Bambou jointes à des branches d'ofier. Ces paniers ont une toife \& demi de longueur \& reffemblent à nos nalfes. Ils s'en fervent, quand l' eau monte plus haut, qu'à l'ordinaire. Ils les mettent le long du rivage, mais j]s laiffent 


\section{DE L'ECONOMIE RURALE}

des ouvertures aux deux bouts du rang de paniers. C'eft là qu'ils fe tiennent tranquilles avec leurs fampanes ou barques, afin que le poiflon, qui cherche le rivage, puiffe y entrer librement. D'abord qu'il y a paffé, il trouve un rang de paniers de Bambou, qui font dilpofés à la traverfe vers le rivage, \& qui lui défendent la fortie. Dès que l'eau commence à s'écouler, ils ferment l'efpace qu'ils avoient laiffé ouvert, avec de pareils paniers. Quand l'eau s'eft entièrement écoulée, ils entrent en dedans de l'enceinte pour ramaffer les poiffons. Ils fe fervent aufi d'un filet flottant attaché entre deux barques avec lequel ils fe promenent pendant le flux, \& prennent les troupes de poiffons qu'ils rencontrent.

Ils fe fervent également d'un grand filet, attaché entre deux bâtons de Bambou, avec lequel ils pêchent, auffi bien dans leurs voyages par mer qu' au milieu de la riviere.

Ils attachent des vers \& des crabes aux hameçons, dont ils prennent des anguilles \&

- d'autres petits poiffons. Ils fe fervent auffi d'une efpèce de Sampanes, qui font longues \& baffes \& qui ont des planches teintes en blanc aux côtés. Ils entretiennent un petit feu dans ces fampanes pendant la nuit. Les poiffons que la lueur du feu atrire, fautent dans la Sampane. Ces Sampanes font proprement conftruites pour une efpèce de poiffo..3: qu'ils appellent Mulottes. 
Ils pêchent beaucoup avec le filet \& l'hamecon entre les brifans \& fur le rivaga, \& prennent quantité de poiffons, qu' ils falent ou qu'ils sèchent pour les vendre dans les villes \& villages voifins.

Parmi la grande variété de poifions, il y en a qui reffemblent à des poiffons connus chés nous, comme les carpes, les perches \&c.; mais je ne puis pas dire fi ce fout les mêmes efpéces. Ceux que je connois avec certitude, font les anguilles, les crabes, les civades, les huitres, les moules $\&$ les homards. De ces derniers on en prend de très grands dans les écueils près de Macao. Ils brulent les coquilles pour en faire de la chaux \& ils fe fervent des plus lar. ges, pour en couvrir leurs maifons au liou de tuiles. 
7

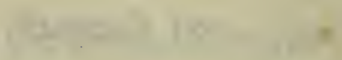

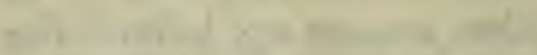

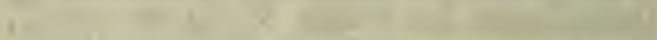

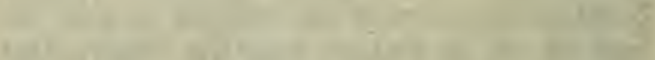

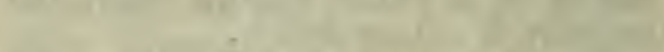

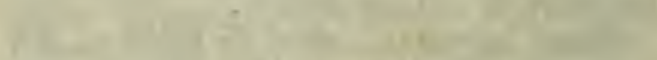

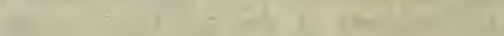
-

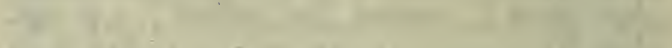

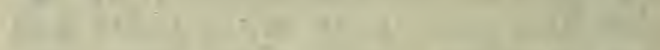

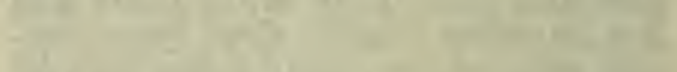

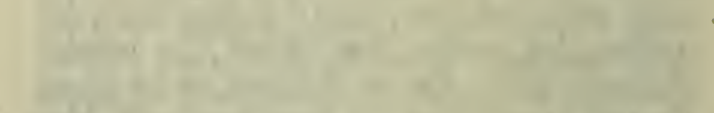

)
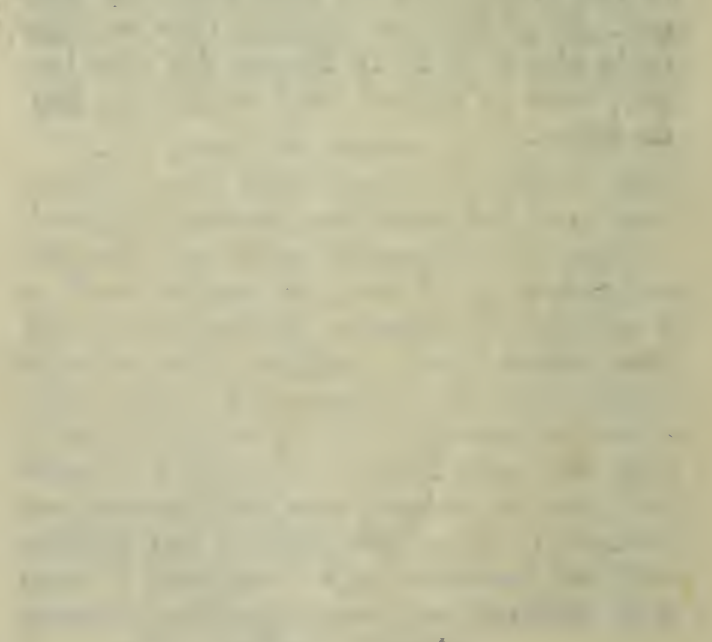

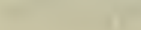

4 
1

.

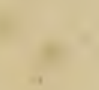

.

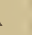





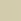

J.

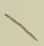

$-1-1-1$ 


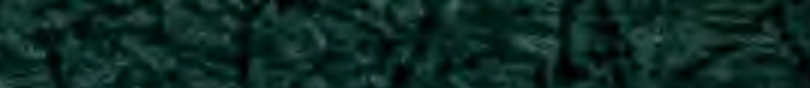

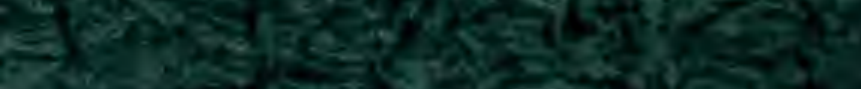

1.

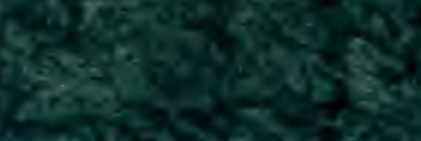

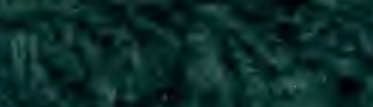

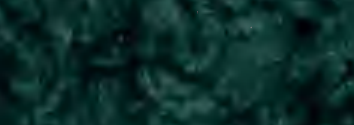<smiles>C1CCC(C2CCCCC2)CC1</smiles>

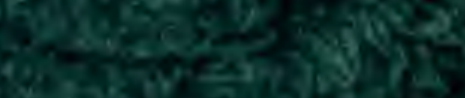

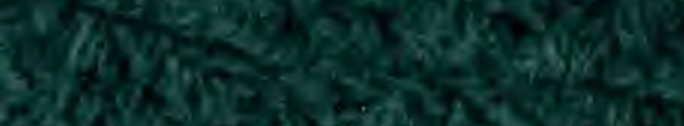

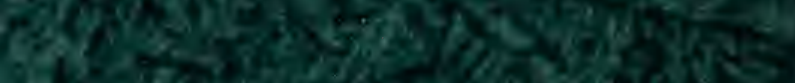

if

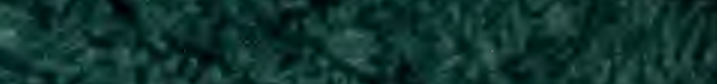

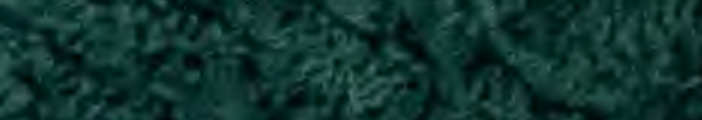

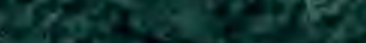

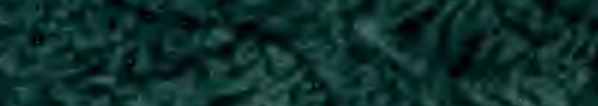

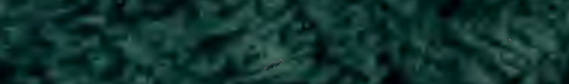

S.

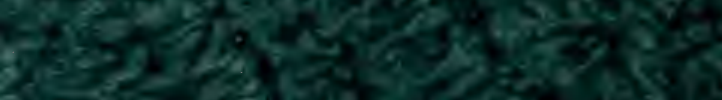
(a)

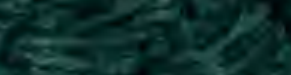

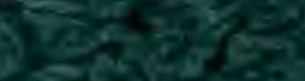

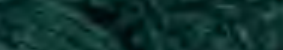

$x^{2}+2 x+2$

Sy.

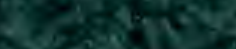

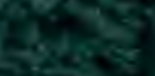

$x^{2}+1$

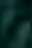

